\title{
One-class land-cover classification using MaxEnt: the effect of modelling parameterization on classification accuracy
}

\author{
Ignacio C Fernández ${ }^{\text {Corresp., } 1}$, Narkis S Morales ${ }^{\text {Corresp. } 1}$ \\ ${ }^{1}$ Centro de Modelación y Monitoreo de Ecosistemas, Facultad de Ciencias, Universidad Mayor, Santiago, Chile \\ Corresponding Authors: Ignacio C Fernández, Narkis S Morales \\ Email address: ignacio.fernandez@umayor.cl, narkis.morales@umayor.cl
}

Multiple-class land-cover classification approaches can be inefficient when the main goal is to classify only one or a few classes. Under this scenario one-class classification algorithms could be a more efficient alternative. Currently there are several algorithms that can fulfil this task, MaxEnt being one of the most promising. However, there is scarce information regarding parametrization for performing land-cover classification using MaxEnt. In this study we aimed to understand how MaxEnt parameterization affects the classification accuracy of four different land-covers (i.e. built-up, irrigated grass, evergreen trees and deciduous trees) in the city of Santiago de Chile. We also evaluated if MaxEnt manual parameterization outperforms classification results obtained when using MaxEnt default parameters setting. To accomplish our objectives, we generated a set of 25,344 classification maps (i.e. 6,336 for each assessed land-cover), which are based on all the potential combination of 12 different classes of features restrictions, four regularization multipliers, four different sample sizes, three training/testing proportions, and 11 thresholds for generating the binary maps. Our results showed that with a good parameterization, MaxEnt can effectively classify different land covers with kappa values ranging from 0.68 for deciduous trees to 0.89 for irrigated grass. However, the accuracy of classification results is highly influenced by the type of land-cover being classified. Simpler models produced good classification outcomes for homogenous land-covers, but not for heterogeneous covers, where complex models provided better outcomes. In general, manual parameterization improves the accuracy of classification results, but this improvement will depend on the threshold used to generate the binary map. In fact, threshold selection showed to be the most relevant factor impacting the four land-cover classification accuracies. The number of sampling points for training the model also has a positive effect on classification results. However, this effect followed a logarithmic distribution, showing an improvement of kappa values when increasing the sampling from 40 to 60 points, but showing only a marginal effect if more than 60 sampling points are used. In light of these results, we suggest testing different parametrization and thresholds 
until satisfactory kappa or other accuracy metrics are achieved. Our results highlight the huge potential that MaxEnt has a as a tool for one-class classification, but a good understanding of the software settings and model parameterization is needed to obtain reliable results. 
One-class land-cover classification using MaxEnt: the effect of modelling parameterization on classification accuracy

Ignacio C. Fernández ${ }^{1^{*}} \&$ Narkis S. Morales ${ }^{1^{*}}$

${ }^{1}$ Centro de Modelación y Monitoreo de Ecosistemas, Facultad de Ciencias, Universidad Mayor, Santiago, Chile.

*Corresponding authors:

Ignacio C. Fernández \& Narkis S. Morales

José Toribio Medina 29, Región Metropolitana, Santiago, 8340589, Chile

Email address: ignacio.fernandez@umayor.cl, narkis.morales@umayor.cl

\section{Abstract}

1 Multiple-class land-cover classification approaches can be inefficient when the main goal is to

2 classify only one or a few classes. Under this scenario one-class classification algorithms could

3 be a more efficient alternative. Currently there are several algorithms that can fulfil this task,

4 MaxEnt being one of the most promising. However, there is scarce information regarding

5 parametrization for performing land-cover classification using MaxEnt. In this study we aimed

6 to understand how MaxEnt parameterization affects the classification accuracy of four different

7 land-covers (i.e. built-up, irrigated grass, evergreen trees and deciduous trees) in the city of

8 Santiago de Chile. We also evaluated if MaxEnt manual parameterization outperforms

9 classification results obtained when using MaxEnt default parameters setting. To accomplish

10 our objectives, we generated a set of 25,344 classification maps (i.e. 6,336 for each assessed 
11 land-cover), which are based on all the potential combination of 12 different classes of features

12

13

14

restrictions, four regularization multipliers, four different sample sizes, three training/testing proportions, and 11 thresholds for generating the binary maps. Our results showed that with a good parameterization, MaxEnt can effectively classify different land covers with kappa values ranging from 0.68 for deciduous trees to 0.89 for irrigated grass. However, the accuracy of classification results is highly influenced by the type of land-cover being classified. Simpler models produce good classification outcomes for homogenous land-covers, but not for heterogeneous covers, where complex models provided better outcomes. In general, manual parameterization improves the accuracy of classification results, but this improvement will depend on the threshold used to generate the binary map. In fact, threshold selection showed to be the most relevant factor impacting the four land-cover classification accuracies. The number of sampling points for training the model also has a positive effect on classification results. However, this effect followed a logarithmic distribution, showing an improvement of kappa values when increasing the sampling from 40 to 60 points, but showing only a marginal effect if more than 60 sampling points are used. In light of these results, we suggest testing different parametrization and thresholds until satisfactory kappa or other accuracy metrics are achieved. Our results highlight the huge potential that MaxEnt has a as a tool for one-class classification, but a good understanding of the software settings and model parameterization is needed to obtain reliable results. 


\section{Introduction}

Land-cover analysis has become a fundamental approach for understanding, modelling and monitoring the spatio-temporal patterns of ecosystems worldwide (Foley et al., 2005; Ellis et al., 2010; Pielke et al., 2011). Land-cover analysis relies on a variety of land classification techniques, mostly based on computational algorithms, that commonly use remote sensing images as a main input for discriminating among different land-cover classes (Rogan and Chen, 2003; Srivastava et al., 2012). These classification techniques are aimed at translating the data contained in the images into usable information, such as land-cover maps. However, as in any modelling procedures, the quality and usefulness of the final outcome will depend on the particular approach used for discriminating among classes of interest (Lu and Weng, 2007). One of the most common techniques for land-cover classification is based on the Maximum Likelihood (ML) algorithm, which is a supervised parametric pixel-based algorithm that is available in most of current GIS or GIS-capable platforms, such as QGIS, ArcGIS and R.

The ML classification assumes that pixel values from a given layer will be normally distributed, therefore each pixel can be classified into a given class based on the maximum likelihood to pertain to the probability density function generated by the samples from that class (Strahler, 1980). Nevertheless, because ML is based on parametric statics, it has shown to be outperformed by newer non-parametric algorithms that do not require the assumption of any a-priori particular distribution or number of parameters, such as Neural Network and Support Vector Machines (Khatami, Mountrakis and Stehman, 2016). Also, ML-based classifications often require to train the algorithm on every single class covering the study area, implying that 
55

56

57

58

59

60

61

62

63

64

65

66

67

many times resources and efforts will be placed on classifying classes that are not relevant for the study objectives (Sanchez-Hernandez, Boyd and Foody, 2007; Li and Guo, 2010; Deng et al., 2018). In fact, multi-class oriented classifiers, such as ML, may attempt to optimize the classification accuracy of all cover classes rather than focusing on increasing the accuracy of the target class (Sanchez-Hernandez, Boyd and Foody, 2007). Therefore, the use of one-class classification algorithms could provide a more efficient alternative than multi-classes approaches when the objective is classifying only one or a few classes of interest (Deng et al., 2018).

Among one-class classification algorithms, MaxEnt is probably one of the most used nowadays. MaxEnt is a maximum entropy algorithm-based software that was originally developed to be used for species distribution modelling (Phillips and Dudík, 2008; Elith et al., 2011). Nevertheless, its built-in algorithm can be easily used for one-class classification of a variety of land-use or -cover types. For example, MaxEnt has been used for mapping urban land-use classes in California (Li and Guo, 2010), urban land in China (Lin et al., 2014), cover of invasive plant species in Colorado (Evangelista et al., 2009) and California (Skowronek, Asner and Feilhauer, 2017), and conservation habitats (Stenzel et al., 2014) and raised bogs in Southern Germany (Mack et al., 2016). The increasing popularity of MaxEnt for one-class landcover classification is probably due to the fact that is a freely available software (https://biodiversityinformatics.amnh.org/open_source/maxent), has shown as good or better performance than other one-class classification methods (e.g. Li \& Guo, 2010, Mack et al., 2016), and comes with default parameters settings that facilitates its operation (Mack and Waske, 2017). 
Nevertheless, while the availability of predefined default parameters may have

78

79

80

promoted MaxEnt massification, the use of this feature can also result in suboptimal land-cover classification outcomes. For example, recent studies from species distribution modelling show that MaxEnt default parameters seldom generate optimal outcomes (Shcheglovitova and Anderson, 2013; Syfert, Smith and Coomes, 2013; Morales, Fernández and Baca-González, 2017). In relation of using MaxEnt default parameter for land-cover classification, the few published studies of which we are aware, also report that MaxEnt tend to produce better classification outcomes when users select the optimal parameters (Mack and Waske, 2017; Skowronek et al., 2018).

There are several default parameters that are modifiable in MaxEnt. The two main modifiable parameters are feature classes (FC) and regularization multiplier (RM). FC corresponds to a set of mathematical transformation of the different covariates used in the model, while RM refers to a numerical parameter that reduces or increases the smoothness of the model (Elith et al., 2011; Merow, Smith and Silander, 2013). Other additional settings, such as sample size (SS), test percentage (TP) and binary threshold (BT), could also influence the final classification outcome. SS refers to the number of samples used for building the model. Although MaxEnt seems to be only slightly sensitive to sample size, larger sample sizes has shown to produce better modeling results (Wisz et al., 2008). TP refers to the proportion (or percentage) of the sample size used for testing the model, which will be the inverse of the proportion used for training. Therefore, TP can affect the model results by indirectly modifying the model sample size. BT corresponds to the specific threshold value used for transforming the continuous probabilistic outputs values into a binary (positive/negative) classification results. 
99 The chosen threshold will largely affect the classification results, nevertheless finding an

100 optimal BT for land-cover classification is still one of the main challenges for using MaxEnt as a

101 one-class classification algorithm (Mack et al., 2016; Mack and Waske, 2017).

102 Even though MaxEnt has shown promising results as a tool for one-class classification,

103 there is still a big knowledge gap regarding how classification results are affected by the

104 parameter settings used for modeling. Thus, in this work we aim to: (1) assess the effects of five

105 different parameter settings (i.e. FC, RM, SS, TP, BT) combinations on MaxEnt-based land-cover

106 classification outcomes; (2) evaluate if these effects depend on the land-cover under analysis;

107 (3) identify potential best parameter setting combination for different land-cover classes and

108 compare its classification accuracy with the outcomes of a default parameter setting

109 classification.

\section{Material and Methods}

\section{Study area}

We developed our study within the metropolitan area of Santiago, Chile $\left(33.4489^{\circ} \mathrm{S}, 70.6693^{\circ}\right.$

W). Santiago is the capital and largest urban area of Chile, harboring an estimated population of

1175.3 million inhabitants (Instituto Nacional de Estadísticas, 2018). The urban area covers a

118 surface of $875 \mathrm{~km}^{2}$ (de la Barrera and Henríquez, 2017), with an elevation ranging from 450 to

$1191000 \mathrm{~m}$ above sea level. The climate within the study area is classified as Mediterranean, with 
121 period during summer (Luebert and Pliscoff, 2006). Historical annual precipitations average

$122 \sim 340 \mathrm{~mm}$, with more than 88\% falling from May to September, and less than 2\% precipitates

123 during the three warmest months (December to February). Seasonal variations in temperatures

124 and precipitation generate noticeable phenological changes in Santiago's urban vegetation,

125 making it feasible to discriminate among different vegetation types from airborne or

126 spaceborne images (Fernandez and De la Barrera, 2018).

127

\section{Remote sensing layers}

To perform the land-cover classification we used Sentinel-2 mission satellite images with 0\% cloud cover, representing vegetation conditions of summer (March 06, 2016) and winter (August 02, 2016) for the study area. To increase the resolution of our analysis, from the set of summer and winter Sentinel-2 images, we only used bands with a native resolution of 10m/pixel (i.e. red, green, blue, NIR). Based on these bands, we also calculated the Normalized Difference Vegetation Index (NDVI) for summer and winter. We also generated an additional layer based on the arithmetic difference between the summer and winter NDVI (i.e. seasonal difference of NDVI). Even though MaxEnt is regarded to be only slightly sensitive to potential over-parameterization issues (Warren and Seifert, 2011), it is recommended to minimize correlation among predictor variables (Merow, Smith and Silander, 2013). Therefore, we

140 performed a Pearson correlation analysis for the 11 layers (i.e. red, green, blue, and NIR, for 141 summer and winter, and the three NDVI layers), and we kept only those layers with correlations 142 smaller than 0.8. After this analysis we ended up with seven layers: Red (summer/winter), NIR 
143 (summer/winter), NDVI (summer/winter) and the seasonal difference of NDVI. All remote-

144 sensing layers procedures were done in QGIS 2.18 Las Palmas (www.qgis.org).

\section{Land-cover classification (Maxent modelling)}

We assessed Maxent one-class classification performance by focusing on four main land-cover

types present in Santiago: (1) Built-up infrastructure, represented by roads and other paved

structures, houses, buildings and commercial infrastructure. (2) Irrigated grass, mostly

associated to private and public lawns, sport fields and other summer irrigated grasses. (3)

Evergreen trees, related to native and exotic urban shrubs and trees with all-year around leaves and photosynthetic activity. (4) Deciduous trees, mostly winter deciduous exotic shrubs and trees planted on residential gardens, streets and urban parks.

To perform the classification modelling we started by selecting 100 ground-true points

for each land-cover class by using very-high resolution images $(<1 \mathrm{~m} /$ pixel) available through

Google Earth. Because potential spatial mismatches between Google and Sentinel images due to georeferencing and resolution differences, we only selected points that were within a $5 \mathrm{~m}$ radius of the type of land-cover under analysis (i.e. minimum size 1 pixel). Each set of points was doubled checked independently by each author. To assess the effect of MaxEnt parametrization on land-cover classification, we generated a set of 25,344 classification maps 
165 of lineal $(\mathrm{L})$, hinge $(\mathrm{H})$, quadratic $(\mathrm{Q})$, threshold $(\mathrm{T})$ and product $(\mathrm{P})$. We used all the

166 computational possible combinations of these features. Second, four regularization multipliers

167 (i.e. 0.25, 1, 3 and 5). Third, four different sample sizes (i.e. 40, 60, 80 and 100). For the later,

168 samples smaller than 100 were randomly sampled from the original 100 sampling points.

169 Fourth, three different percentages of testing proportion to validate the model (i.e. 10, 30, 50).

170 Fifth, 11 binary thresholds for generating the final binary classification maps: Fixed cumulative

171 value 1 threshold (FC1); fixed cumulative value 5 threshold (FC5); fixed cumulative value 10

172 threshold (FC10); 10 percentile training presence threshold (10PTP); balance training omission,

173 predicted area and threshold value threshold (BTOPA); equate entropy of thresholded and

174 original distributions threshold (EETOD); equal test sensitivity and specificity threshold (ETeSS);

175 equal training sensitivity and specificity threshold (ETrSS); maximum test sensitivity plus

176 specificity threshold (MTeSPS); minimum training presence threshold (MTP); maximum training

177 sensitivity plus specificity threshold (MTrSPS) (Table 1). All MaxEnt models were run using the

178 random seed feature and replicated 10 times, and we used the average values of these

179 replicates as the modeling outcomes. Background points were set to default (maximum 10000

180 points). All modelling procedures were performed using MaxEnt 3.4.1

181 (https://biodiversityinformatics.amnh.org/open_source/maxent). Application of thresholds and 182 generation of final binary maps were done in R version 3.5.1 (www.r-project.org). 
186 We evaluated the accuracy of the classification result by estimating the kappa coefficient. To 187 calculate kappa, we randomly sampled 1000 points within a quadrant of $16 \times 16 \mathrm{~km}$, which is 188 the largest square that can be drawn within the convoluted shape of Santiago. We decided to use this quadrant to avoid potential undesirable effects of choosing sampling points outside Santiago's urbanized area and to reduce the size of files to increase the efficiency of the computational processes. Each of these 1000 points were visually classified into built-up, irrigated grass, evergreen trees, deciduous trees and "other class" by using very-high resolution images through Google Earth. If a sampling point fell on mixed land-cover (e.g. transition between two or more land covers) we moved the point to the closest single-land cover surface representing the dominant land-cover from the original location. We considered mixed landcovers all points falling in locations with more than one land-cover within a $5 \mathrm{~m}$ radius. All points were double checked by the authors. We then used the 1000 sampling points to build four different testing layers (one for each assessed land-cover) based on positive and negative labels for each land-cover. We used these testing layers to calculate the kappa coefficient for each classification result based on the proportion of true positive, false positive, true negative and false negative, following the formula shown in Fielding and Bell (1997).

We compared the classification accuracy of the different combinations through comparison of differences on data distribution, interquartile and median values. All classification accuracy procedures and graphs were done in $\mathrm{R}$ version 3.5.1. 
209 We defined the best combination of parameter for each land-cover type by looking at the 210 kappa's median values of the violin-boxplots of feature classes, regularization multipliers,

211 sample size and test percentage (See Figs. 1,2,3 and 4). Based on this result, we decided to use

212 a SS of 100 sampling points and a TP of $10 \%$ for all classification models (i.e. default and best

213 parameter settings), only modifying those parameters directly related to the modeling process,

214 which are the FC and RM. Therefore, for the default parameter setting models we used the 215 "autofeature" FC with a RM of 1, whereas for the best parameter setting we used the FC and

216 RM showing the highest median kappa for each land-cover type (See Figs. 1 and 2). Using these

217 parameters, we ran 30 replicates for each classification model in MaxEnt. To generate the final

218 binary maps in $\mathrm{R}$, we did not select a specific binary threshold because we did not know the

219 effect of this parameter on the default setting models results. Instead, we compared modeling

220 results by using all the BT provided in MaxEnt. We used a conventional student t-test to

221 evaluate statistically significant differences between the default and best parameter settings

222 for each land-cover type and binary threshold.

223

224 3. Results

225

Parameterization effects on classification accuracy

227

Results show that changing the feature classes can have an important effect on the final land- 
230 being classified (Fig. 1). For example, while for built-up land cover the $L$ and $Q$ features

231 produced the highest kappa (measured as kappa's median value), these same features

232 produced the poorest results for the three vegetated covers (i.e. irrigated grass, evergreen

233 trees and deciduous trees). Furthermore, the FC for which the kappa median was largest,

234 fluctuated for the four land-covers: L for built-up, LQPTH for irrigated grass, QP for evergreen

235 trees and QH for deciduous trees, with median kappa's of 0.695, 0.693, 0.577 and 0.528,

236 respectively. There are also important differences in the variability (i.e. density distribution) of

237 kappa results associated to the different FC. For example, based on the differences between

238 the first and third quartile, built-up, evergreen trees and deciduous trees covers show the

239 largest dispersion of kappa values for $T, Q$, and $T Q$, but for irrigated grass all FC show very large

240 dispersion, except for L and Q (See Fig. 1).

241 Modifying the regularization multiplier could also have important effect on classification

242 results, but as our results show, these effects could considerably differ depending on the land-

243 cover type being classified (Fig. 2). While for built-up, increasing the RM from 0.25 to 1 seems

244 to slightly improve classification results, the opposite pattern is observed for the three

245 vegetated covers. In addition, while further increases (> 1) in RM do not improve classification

246 outcomes for built-up cover, they do improve the classification for vegetated covers, as is

247 shown by the consistent increase in kappa with larger RM. Among the three vegetated covers

248 assessed in this work, irrigated grass cover showed by far the largest response to changes in RM

249 (Fig. 2).

$250 \quad$ Changes in sample size show only a slight effect on classification results (Fig. 3).

251 Although there are some differences between the four assessed land-covers, there is a 
252 consistent pattern showing that the lowest SS (i.e. 40 samples) generated the poorest

253 classification results. For all assessed land covers, increasing the SS from 40 to 60 improved the

254 classification. However, results show that increases in SS above 60 samples do not necessarily

255 generate large improvements on classification results (Fig. 3).

256 Changing the test percentage for validating the model has only weak effects on

257 classification results. In fact, results show that for built-up cover this setting seems to not affect

258 the median value of kappa nor the kappa's median distribution, whereas for evergreen trees

259 and deciduous trees it is observable a slight, but consistent decrease in classification results as

260 TP increase to 50\% (Fig. 4). The larger effect of TP is shown for irrigated grass cover, which is

261 consistent with the results shown for changes in the SS setting.

The selection of binary threshold values is the setting that had the largest effects on

classification results among the five assessed settings (Fig. 5). Using one BT instead of another

could greatly affect the classification results. For example, depending on the selected threshold,

kappa's median for irrigated grass cover could range from 0.134 to 0.811 (Fig. 5b). The effect of

BT on classification shows similar patterns for irrigated grass, evergreen trees and deciduous

trees, but not for built-up cover. For instance, while for built-up cover the FC1 threshold

generated one of the best classification results ( $k a p p a$ 's median $=0.766)$, for the three

produced the best classification results for irrigated grass and evergreen trees, and the second

271 best for deciduous trees, but produced poor classification results for built-up cover (Fig. 5). 
274 The comparison of the models produced using the default parameter setting for FC and RM 275 with those using the identified best parameters (defined as FC and RM having the largest kappa 276 median for each land-cover type, i.e. $F C=L, R M=1$ for built-up; $F C=L Q P T H, R M=0.25$ for irrigated 277 grass; $\mathrm{FC}=\mathrm{QP}, \mathrm{RM}=0.25$ for evergreen trees; $\mathrm{FC}=\mathrm{QH}, \mathrm{RM}=0.25$ for deciduous trees; See Figs. 1 278 and 2) show that the identified best parameter setting does not always outperform the

279 classification accuracy of the default setting model (Fig. 6). Considering the four assessed landcover types and the 11 thresholds, the best parameters outperformed the default parameters in 23 out of the 44 classification result comparisons $(53,3 \%)$, while the identified best parameter was outperformed by the default parameter in 11 cases (25,0\%). In 10 comparisons $(22,7 \%)$ there were no statistical differences between both parameters settings (Fig. 6). The difference in classification results between the default and identified best parameters is highly dependent on the land-cover under analysis and the threshold used for generating the binary maps. For example, for evergreen trees, in 9 out of 11 comparison the best parameter setting outperformed the default model and for no threshold the default model resulted in better classification. On the other hand, for irrigated grass the default model outperformed the identified best parameter setting in five out of 11 comparisons, while the opposite results were obtained 6 times (Fig. 6). In general, BT selection has a much larger effect than parameter settings in classification results.

\section{Discussion}


295 Results from our work add to previous studies showing the potential of MaxEnt to be used as a

296 one-class land-cover classification method (e.g. Li and Guo, 2010; Amici, 2011; Lin et al., 2014;

297 Stenzel et al., 2014), but also draw attention to the importance of selecting best parameters

298 combinations to achieve good classification accuracies (Mack and Waske, 2017). While our

299 main objective was to understand how MaxEnt parameterization may affect classification

300 results, we were also able to test the capability of MaxEnt for one-class land-cover

301 classification, which has never been tested in an entire urban area the size of Santiago.

302 Our classification accuracy shows kappa values above 0.6 for all the assessed land-

303 covers -and even above 0.8 for built-up and irrigated grass. These kappa values are comparable

304 to those obtained by Li and Guo (2010) using Maxent to classify similar land-covers in a urban

305 plot in a residential area of California, and also to those obtained by Deng et al. (2018) using

306 other state of the art classification algorithms to classify land covers in a similar urban setting in

307 California. Thus, results from our study highlight the potential of MaxEnt and its built-in

308 maximum entropy algorithm to be used for one-class classification in complex spatially

309 heterogeneous settings, such as urban areas.

310

311

Parameterization effects on classification accuracy

312

313 Results from our work show that MaxEnt parameterization plays an important role on land-

314 cover classification accuracy, and therefore is highly relevant for achieving good classification

315 results. However, based in our results, is not clear if there are some specific feature classes that

316 can provide higher quality classifications. On the contrary, it seems that the optimal feature 
317 classes to use will depend on the intrinsic spatial variability of the layers used to build the

318 models. For example, in our study, the $L$ and Q (i.e. lineal and quadratic) feature classes

319 generate among the best classification accuracy for built-up, but the poorest results for

320 vegetated covers. This result could be explained because the $Q$ feature constrains the variation

321 of the predictor variable to that of the sampling points of each layer (See Elith et al., 2011;

322 Merow, Smith and Silander, 2013 for an explanation of MaxEnt features). Thus, the Q feature

323 may differentially affect the specificity of the resulting model depending on the true variance of

324 the population, benefiting classification results of spatially homogenous covers, such as built-

325 up, but weakening results for more heterogeneous covers, such as irrigated grass. A similar

326 interpretation could be made for the $L$ feature, as this feature simply uses the mean of the

327 sampling points to estimate the conditions making a pixel suitable to be classified into a given

328 class. These results suggest that MaxEnt models based on simpler parameter combination could

329 be well suited for classifying land-covers with more homogenous reflectance behaviors, such as

330 built-up areas, while models of higher complexity would be needed for vegetated or other

331 more heterogeneous areas. This could be further supported by the results of the regularization

332 multiplier, that show that for vegetated land-covers accuracy increases if regularization

333 multiplier is reduced to fit more complex models, whereas built-up classification accuracy

334 benefits from larger regularization that smooths out the model fit.

335 While sample size may not be directly considered a model parameter, it is can have a

336 direct effect on MaxEnt behavior, particularly if default features are used. In fact, MaxEnt will

337 restrict the use of potential feature classes depending of the number of sampling points (Elith

338 et al., 2011; Merow, Smith and Silander, 2013), therefore models generated with a small 
number of samples will be restricted in their complexity. This may explain why classification of sampling points used for training the model. Nevertheless, for the four land-covers, an effective sampling size larger than $\sim 80$ seems to be optimal, which is consistent with the sampling size threshold at which MaxEnt is set to use all potential FC (Elith et al., 2011). the classified maps is perhaps the most crucial factor related to the accuracy of the final classification outcome. This threshold is not a MaxEnt modeling parameter, but rather $a$ potential thresholds (the 11 used in this study), the final decision on what threshold to use will mostly depend on the researcher or group of researchers performing the classification. threshold to maximize the classification accuracy using MaxEnt (Li and Guo, 2010; Mack et al., 2016; Mack and Waske, 2017), and based in our results it seems that this quest is still open. In fact, none of the tested thresholds performed well for all the assessed land-covers, implying that users will need to explore a set of thresholds to find one that produce optimal classification accuracy for each land-cover.

Default parameter vs best parameters setting classification accuracy

There is increasing evidence that using best, instead of default parameters, could improve the 360 predictability of species distribution models produced through MaxEnt (Shcheglovitova and 
361 Anderson, 2013; Morales, Fernández and Baca-González, 2017), and similar results have

362 recently been found when using MaxEnt for land-cover classification (Mack and Waske, 2017).

363 However, our results show that although in general best parameters outperformed the default

364 parameters, for some land-covers and thresholds, default parameters can produce similar or

365 even better classification results than the identified best parameters. Technically this sounds

366 illogical, because a manually parameterized model can theoretically use the same parameters

367 of the default model, and therefore if best model parameters are found, classification results

368 should be at least equally good as the one obtained by the default model. Nevertheless, finding

369 the best parameters combination is still a challenging task (Muscarella et al., 2014). For

370 example, to find the optimal parameters combination for our study, we run thousands of

371 models and selected each parameter based on the median value of resulting kappas, assuming

372 that general central tendencies could be indicative of parameters performances. However, this

373 approach ruled out potential parameters combinations that produced higher kappas (See

374 supplementary material), which may help explaining why in our study for some comparisons

375 the default parameter had better classification accuracies than the best model parameter.

376 An interesting finding from our study was the fact that threshold selection do not only

377 affect the accuracy of classification results (Mack and Waske, 2017), but that a same threshold

378 could differentially impact on the accuracy of different models (Fig. 6a). These findings show

379 that just using the model performance information, such as AUC (Area Under the Curve), for

380 evaluating the accuracy of a land-cover classification outcomes is not advisable.

381

382

Best parametrization practices

Peer] reviewing PDF | (2019:02:35347:1:1:NEW 20 Apr 2019) 
384 In the light of our results and other articles regarding the use of MaxEnt, we would like to highlight the importance of parameterizing MaxEnt for each model individually and avoid the temptation to of using default parameter or previous studies parametrizations (Merow, Smith and Silander, 2013; Mack and Waske, 2017; Morales, Fernández and Baca-González, 2017). As a rule of thumb, a comparison of different models with different parametrization settings must be performed to determine the best model among all the evaluated parameters combinations. Ideally, selection of parameters combination to be tested should not be random, but rather on the specific objectives and land-cover under analysis. For example, for contrasting and homogenous land-cover such as paved areas, using combinations that generate simpler models could be an efficient approach. Whereas for less contrasting and heterogeneous land-covers, such as irrigated grass, it would be recommended to increase the number of tested combinations to include more complex models. There are several techniques available for selecting MaxEnt best models, including jackknife procedures based on the corrected Akaike information criterion (AICC) (Shcheglovitova and Anderson, 2013; Morales, Fernández and BacaGonzález, 2017), correlation analysis (Syfert, Smith and Coomes, 2013), fuzzy kappa statistics (Mestre et al., 2015) and software including specific algorithms for this objective (Warren, Glor and Turelli, 2010; Muscarella et al., 2014). Nevertheless, once the parametrization of the model

401 is done, thresholds to generate binary maps still need to be chosen. As our results show,

402 threshold selection could be one of the most important factors influencing the final 403 classification accuracy. In this regard, we recommend not to use predefined thresholds or 404 thresholds used in previous studies, but instead try a set of different thresholds and select the 
405 one that gives the best outcome. A viable option could be using a set of different thresholds

406 based on cumulative percentiles and select the generated model with the largest classification

407 accuracy (Lin et al., 2014). Based in our results, percentiles between 1 and 10 seems to be a

408 good testing ground, as best classification outcomes tend to be produced by thresholds within

409 this percentile range. Nevertheless, further research is needed to better understand how

410 variation in threshold values affects the accuracy of land-cover classification produced through

411 MaxEnt.

412

413 5. Conclusion

414

415

Our results showed that MaxEnt can be a useful tool for single-class land-cover classification.

However, the classification accuracy heavily depends on land-cover characteristics and parameters used for modelling. Based in these results, we suggest avoiding the temptation of using MaxEnt default parameterization features in heterogeneous layers, as manual parameterization could generate better land-classification accuracy outcomes. In addition, selection of optimal binary thresholds is key for increasing classification accuracy when using binary maps. Nevertheless, for this is still essential to use a classification accuracy metric for testing modelling results. In light of these results, we suggest testing different parametrization settings and thresholds until satisfactory kappa or other accuracy metrics are achieved. Finally,

424 is also important to bear in mind that the reliability of the selected model will be also 425 influenced by the accuracy metric used for classification testing. In this regard, for specific 
426

427

428

429

430

431

432

433

434

435

436

437

438

439

440

441

442

443

444

445

446

447

448

449

450

451

452

453

454

455

456

457

458

459

460

461

462

463

464

465

classification tasks, it would be recommendable to use multiple accuracy metrics against which

to compare the resulting models.

\section{References}

Amici, V. (2011) 'Dealing with vagueness in complex forest landscapes: A soft classification approach through a niche-based distribution model', Ecological Informatics, pp. 371-383. doi: 10.1016/j.ecoinf.2011.07.001.

Deng, X., Li, W., Liu, X., Guo, Q. and Newsam, S. (2018) 'One-class remote sensing classification: One-class vs. Binary classifiers', International Journal of Remote Sensing. Taylor \& Francis, 39(6), pp. 1890-1910. doi: 10.1080/01431161.2017.1416697.

Elith, J., Phillips, S. J., Hastie, T., Dudík, M., Chee, Y. E. and Yates, C. J. (2011) 'A statistical explanation of MaxEnt for ecologists', Diversity and Distributions, 17(1), pp. 43-57. doi: 10.1111/j.1472-4642.2010.00725.x.

Ellis, E. C., Klein Goldewijk, K., Siebert, S., Lightman, D. and Ramankutty, N. (2010)

'Anthropogenic transformation of the biomes, 1700 to 2000', Global Ecology and Biogeography, 19, pp. 589-606. doi: 10.1111/j.1466-8238.2010.00540.x.

Evangelista, P. H., Stohlgren, T. J., Morisette, J. T. and Kumar, S. (2009) 'Mapping invasive tamarisk (Tamarix): A comparison of single-scene and time-series analyses of remotely sensed data', Remote Sensing, pp. 519-533. doi: 10.3390/rs1030519.

Fernandez, I. C. and De la Barrera, F. (2018) 'Biodiversidad urbana, servicios ecosistémicos y planificación ecológica : un enfoque desde la ecología del paisaje', in Figueroa, J. and Lazzoni, I. (eds) Biodiversidad Urbana en Chile: Estado del arte y los desafíos futuros. First. Santiago, Chile: Universidad Central de Chile, pp. 113-146.

Fielding, A. H. and Bell, J. F. (1997) 'A review of methods for the assessment of prediction errors in conservation presence/absence models', Environmental Conservation, 24, pp. 38-49. Foley, J. A., Defries, R., Asner, G. P., Barford, C., Bonan, G., Carpenter, S. R., Chapin, F. S., Coe, M. T., Daily, G. C., Gibbs, H. K., Helkowski, J. H., Holloway, T., Howard, E. a, Kucharik, C. J., Monfreda, C., Patz, J. a, Prentice, I. C., Ramankutty, N. and Snyder, P. K. (2005) 'Global consequences of land use.', Science, 309(570), pp. 570-574. doi: 10.1126/science.1111772. Instituto Nacional de Estadísticas (2018) Población por sexo y grupo de edad, Censo de Población y Vivienda 2017. Available at: https://redatamine.ine.cl/redbin/RpWebEngine.exe/Portal?BASE=CENSO_2017\&lang=esp (Accessed: 9 September 2018).

Khatami, R., Mountrakis, G. and Stehman, S. V. (2016) 'A meta-analysis of remote sensing research on supervised pixel-based land-cover image classification processes: General guidelines for practitioners and future research', Remote Sensing of Environment. Elsevier Inc., 177, pp. 89-100. doi: 10.1016/j.rse.2016.02.028.

de la Barrera, F. and Henríquez, C. (2017) 'Vegetation cover change in growing urban agglomerations in Chile', Ecological Indicators. Elsevier, 81, pp. 265-273. doi: 10.1016/j.ecolind.2017.05.067. 
$466 \mathrm{Li}, \mathrm{W}$. and Guo, Q. (2010) 'A maximum entropy approach to one-class classification of remote

467

468

469

470

471

472

473

474

475

476

477

478

479

480

481

482

483

484

485

486

487

488

489

490

491

492

493

494

495

496

497

498

499

500

501

502

503

504

505

506

507

508

509 sensing imagery', International Journal of Remote Sensing, 31(8), pp. 2227-2235. doi: 10.1080/01431161003702245. Lin, J., Liu, X., Li, K. and Li, X. (2014) 'A maximum entropy method to extract urban land by combining MODIS reflectance, MODIS NDVI, and DMSP-OLS data', International Journal of Remote Sensing, 35(18), pp. 6708-6727. doi: 10.1080/01431161.2014.960623. Lu, D. and Weng, Q. (2007) 'A survey of image classification methods and techniques for improving classification performance', International Journal of Remote Sensing, 28(5), pp. 823870. doi: 10.1080/01431160600746456.

Luebert, F. and Pliscoff, P. (2006) Sinopsis bioclimática y vegetacional de Chile, Biodiversidad. Santiago, Chile: Editorial Universitaria.

Mack, B., Roscher, R., Stenzel, S., Feilhauer, H., Schmidtlein, S. and Waske, B. (2016) 'Mapping raised bogs with an iterative one-class classification approach', ISPRS Journal of Photogrammetry and Remote Sensing, pp. 53-64. doi: 10.1016/j.isprsjprs.2016.07.008. Mack, B. and Waske, B. (2017) 'In-depth comparisons of MaxEnt, biased SVM and one-class SVM for one-class classification of remote sensing data', Remote Sensing Letters. Taylor \& Francis, 8(3), pp. 290-299. doi: 10.1080/2150704X.2016.1265689.

Merow, C., Smith, M. J. and Silander, J. A. (2013) 'A practical guide to MaxEnt for modeling species' distributions: What it does, and why inputs and settings matter', Ecography, 36(10), pp. 1058-1069. doi: 10.1111/j.1600-0587.2013.07872.x.

Mestre, F., Pita, R., Paupério, J., Martins, F. M. S., Alves, P. C., Mira, A. and Beja, P. (2015) 'Combining distribution modelling and non-invasive genetics to improve range shift forecasting', Ecological Modelling. Elsevier B.V., 297, pp. 171-179. doi: 10.1016/j.ecolmodel.2014.11.018.

Morales, N. S., Fernández, I. C. and Baca-González, V. (2017) 'MaxEnt's parameter configuration and small samples: are we paying attention to recommendations? A systematic review', PeerJ, 5, p. e3093. doi: 10.7717/peerj.3093.

Muscarella, R., Galante, P. J., Soley-Guardia, M., Boria, R. A., Kass, J. M., Uriarte, M. and Anderson, R. P. (2014) 'ENMeval: An R package for conducting spatially independent evaluations and estimating optimal model complexity for $<\mathrm{scp}>$ Maxent $</ \mathrm{scp}>$ ecological niche models', Methods in Ecology and Evolution, 5(11), pp. 1198-1205. doi: 10.1111/2041210X.12261.

Phillips, S. and Dudík, M. (2008) 'Modeling of species distributions with Maxent: new extensions and a comprehensive evaluation', Ecography, (31), pp. 161-175. doi: 10.1111/j.2007.09067590.05203.x.

Pielke, R. A., Pitman, A., Niyogi, D., Mahmood, R., McAlpine, C., Hossain, F., Goldewijk, K. K., Nair, U., Betts, R., Fall, S., Reichstein, M., Kabat, P. and de Noblet, N. (2011) 'Land use/land cover changes and climate: Modeling analysis and observational evidence', Wiley Interdisciplinary Reviews: Climate Change, 2(6), pp. 828-850. doi: 10.1002/wcc.144.

Rogan, J. and Chen, D. (2003) 'Remote sensing technology for mapping and monitoring landcover and land-use change', Progress in Planning, 61(61), pp. 301-325. doi: 10.1016/S03059006(03)00066-7.

Sanchez-Hernandez, C., Boyd, D. S. and Foody, G. M. (2007) 'One-class classification for mapping a specific land-cover class: SVDD classification of fenland', IEEE Transactions on

PeerJ reviewing PDF | (2019:02:35347:1:1:NEW 20 Apr 2019) 
510 Geoscience and Remote Sensing, pp. 1061-1072. doi: 10.1109/TGRS.2006.890414.

511 Shcheglovitova, M. and Anderson, R. P. (2013) 'Estimating optimal complexity for ecological

512 niche models: A jackknife approach for species with small sample sizes', Ecological Modelling.

513 Elsevier B.V., 269, pp. 9-17. doi: 10.1016/j.ecolmodel.2013.08.011.

514 Skowronek, S., Asner, G. P. and Feilhauer, H. (2017) 'Performance of one-class classifiers for

515 invasive species mapping using airborne imaging spectroscopy', Ecological Informatics. Elsevier

516 B.V., 37, pp. 66-76. doi: 10.1016/j.ecoinf.2016.11.005.

517 Skowronek, S., Van De Kerchove, R., Rombouts, B., Aerts, R., Ewald, M., Warrie, J., Schiefer, F.,

518 Garzon-Lopez, C., Hattab, T., Honnay, O., Lenoir, J., Rocchini, D., Schmidtlein, S., Somers, B. and

519 Feilhauer, H. (2018) 'Transferability of species distribution models for the detection of an

520 invasive alien bryophyte using imaging spectroscopy data', International Journal of Applied

521 Earth Observation and Geoinformation. Elsevier, 68(February), pp. 61-72. doi:

522 10.1016/j.jag.2018.02.001.

523 Srivastava, P. K., Han, D., Rico-Ramirez, M. A., Bray, M. and Islam, T. (2012) 'Selection of

524 classification techniques for land use/land cover change investigation', Advances in Space

525 Research. COSPAR, 50(9), pp. 1250-1265. doi: 10.1016/j.asr.2012.06.032.

526 Stenzel, S., Feilhauer, H., Mack, B., Metz, A. and Schmidtlein, S. (2014) 'Remote sensing of

527 scattered natura 2000 habitats using a one-class classifier', International Journal of Applied

528 Earth Observation and Geoinformation, pp. 211-217. doi: 10.1016/j.jag.2014.05.012.

529 Strahler, A. H. (1980) 'The use of prior probabilities in maximum likelihood classification of

530 remotely sensed data', Remote Sensing of Environment, 10(2), pp. 135-163. doi:

531 10.1002/erv.2405.

532 Syfert, M. M., Smith, M. J. and Coomes, D. A. (2013) 'The Effects of Sampling Bias and Model

533 Complexity on the Predictive Performance of MaxEnt Species Distribution Models', PLoS ONE,

534 8(2). doi: 10.1371/journal.pone.0055158.

535 Warren, D. L., Glor, R. E. and Turelli, M. (2010) 'ENMTools: a toolbox for comparative studies of 536 environmental niche models', Ecography, 1(September 2009), pp. 607-611. doi:

537 10.1111/j.1600-0587.2009.06142.x.

538 Warren, D. L. and Seifert, S. N. (2011) 'Ecological niche modeling in Maxent : the importance of 539 model complexity and the performance of model selection criteria', Ecological Applications, 540 21(2), pp. 335-342. doi: 10.2307/29779663.

541 Wisz, M. S., Hijmans, R. J., Li, J., Peterson, A. T., Graham, C. H., Guisan, A., Elith, J., Dudík, M., 542 Ferrier, S., Huettmann, F., Leathwick, J. R., Lehmann, A., Lohmann, L., Loiselle, B. A., Manion, G., 543 Moritz, C., Nakamura, M., Nakazawa, Y., Overton, J. M. C., Phillips, S. J., Richardson, K. S., 544 Scachetti-Pereira, R., Schapire, R. E., Soberón, J., Williams, S. E. and Zimmermann, N. E. (2008) 545 'Effects of sample size on the performance of species distribution models', Diversity and 546 Distributions, 14(5), pp. 763-773. doi: 10.1111/j.1472-4642.2008.00482.x. 


\section{Table 1 (on next page)}

Parameters used to generate the land-cover classification results

See methodological section for references to the acronym used in FC and BT columns. 
1

\begin{tabular}{ccccc}
\hline $\begin{array}{c}\text { Feature Class } \\
\text { (FC) }\end{array}$ & $\begin{array}{c}\text { Regul. Multiplier } \\
\text { (RM) }\end{array}$ & $\begin{array}{c}\text { Sample Size } \\
(\text { SS) }\end{array}$ & $\begin{array}{c}\text { Test Percentage } \\
\text { (TP) }\end{array}$ & $\begin{array}{c}\text { Binary Threshold } \\
\text { (BT) }\end{array}$ \\
\hline L & 5.00 & 100 & 50 & FC1 \\
H & 3.00 & 80 & 30 & FC5 \\
Q & 1.00 & 60 & 10 & FC10 \\
T & 0.25 & 40 & & $10 P T P$ \\
LQ & & & & BTOPA \\
PT & & & & EETOD \\
QH & & & & ETeSS \\
QP & & & & ETrSS \\
TQ & & & & MTeSPS \\
LQP & & & MTP \\
LQPT & & & \\
LQPTH & & & \\
\hline
\end{tabular}

Table 1. Parameters used to generate the land-cover classification results. See methodological section for references to the acronym used in FC and BT columns.

2 
Figure 1

Violinplots of the kappa values obtained from the single land-cover maps generated through MaxEnt using the feature classes (FC) shown in the x-axis.

Each violinplot includes the combination of all the other tested parameters (See Table 1 for reference). Red dashed line is the median kappa value for all combinations.
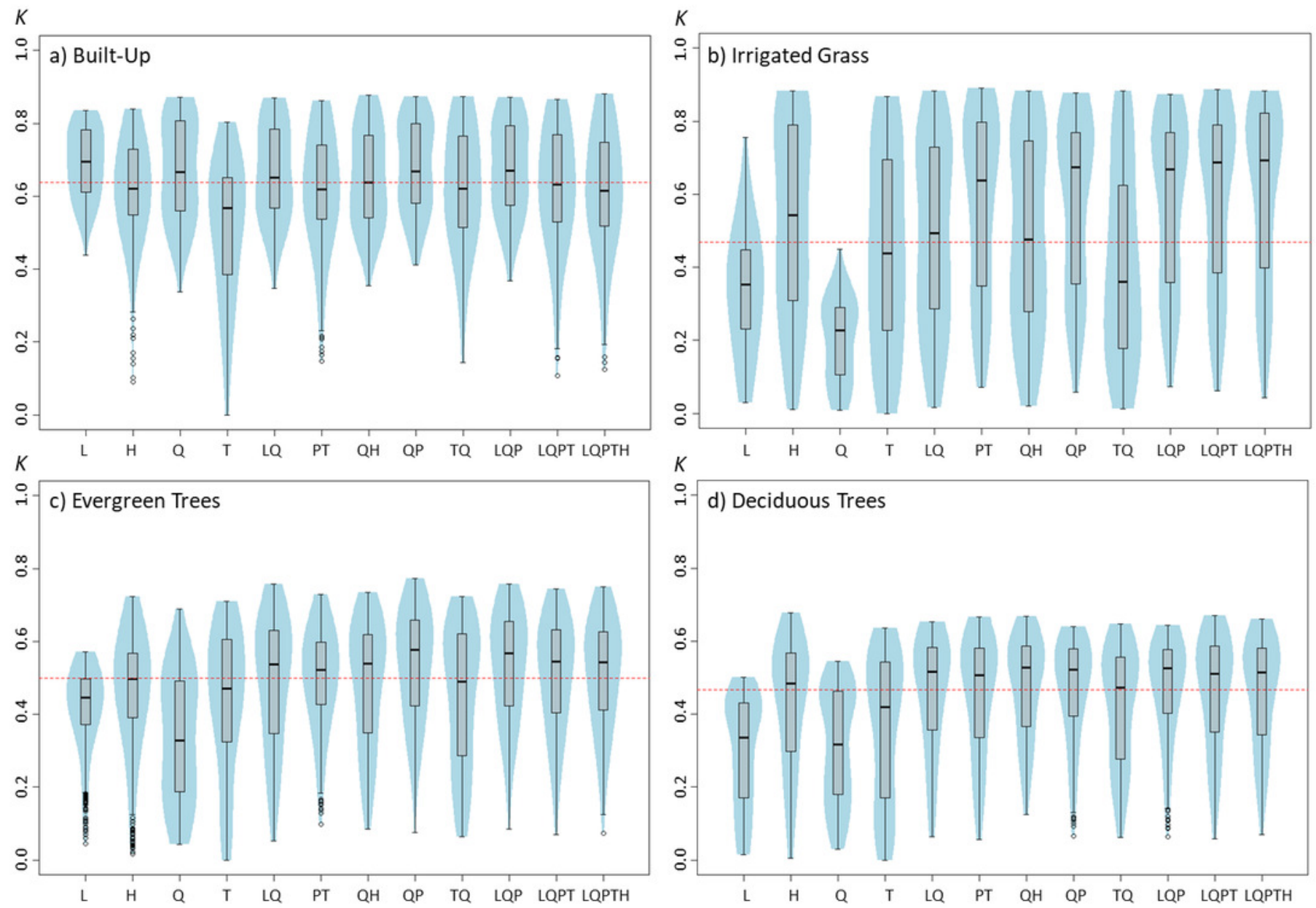
Figure 2

Violinplots of the kappa values obtained from the single land-cover maps generated through MaxEnt using the regularization multipliers (RM) shown in the $x$-axis.

Each violinplot includes the combination of all the other tested parameters (See Table 1 for reference). Red dashed line is the median kappa value for all combinations. 

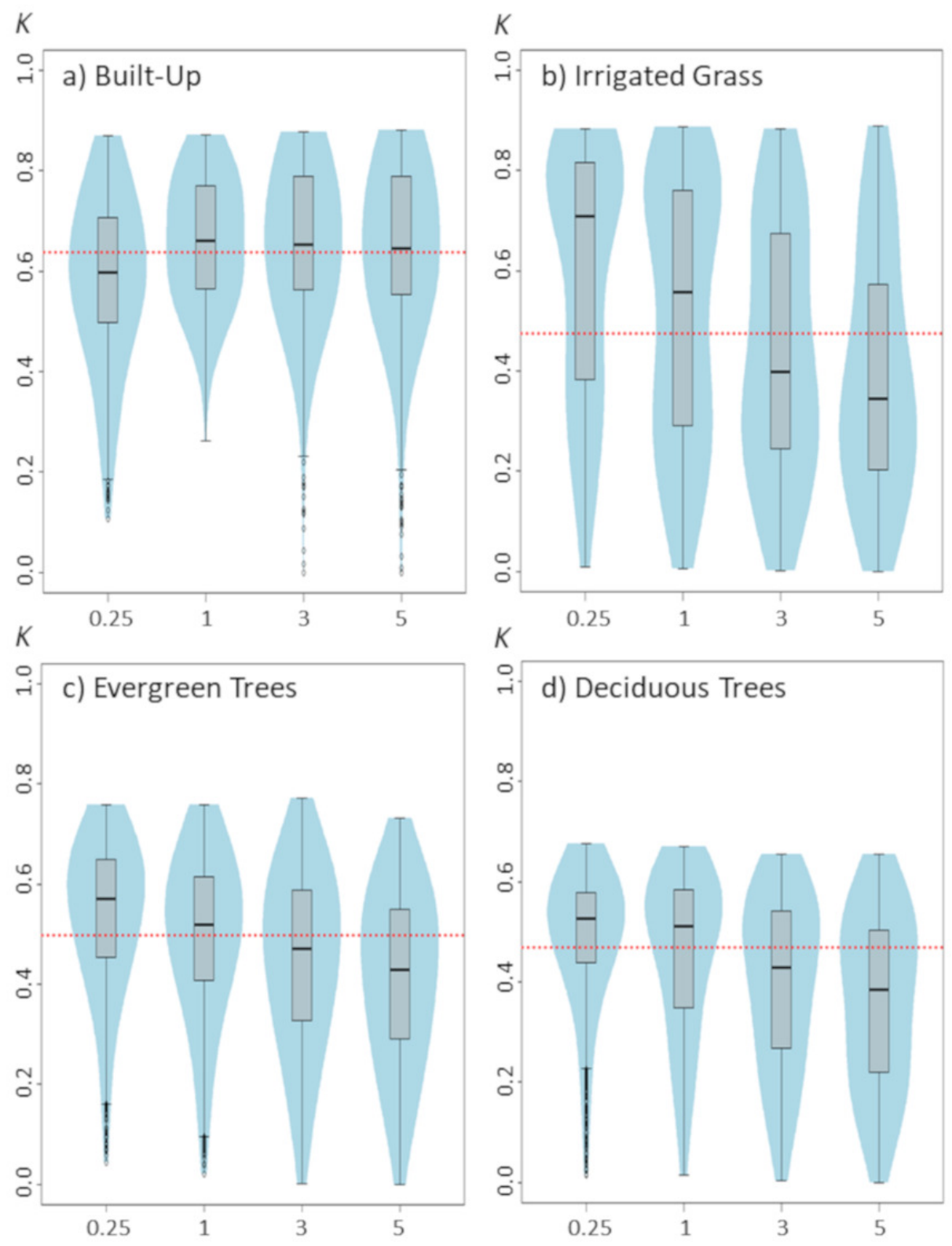
Figure 3

Violinplots of the kappa values obtained from the single land-cover maps generated through MaxEnt using the sample size (SS) shown in the x-axis.

Each violinplot includes the combination of all the other tested parameters (See Table 1 for reference). Red dashed line is the median kappa value for all combinations. 

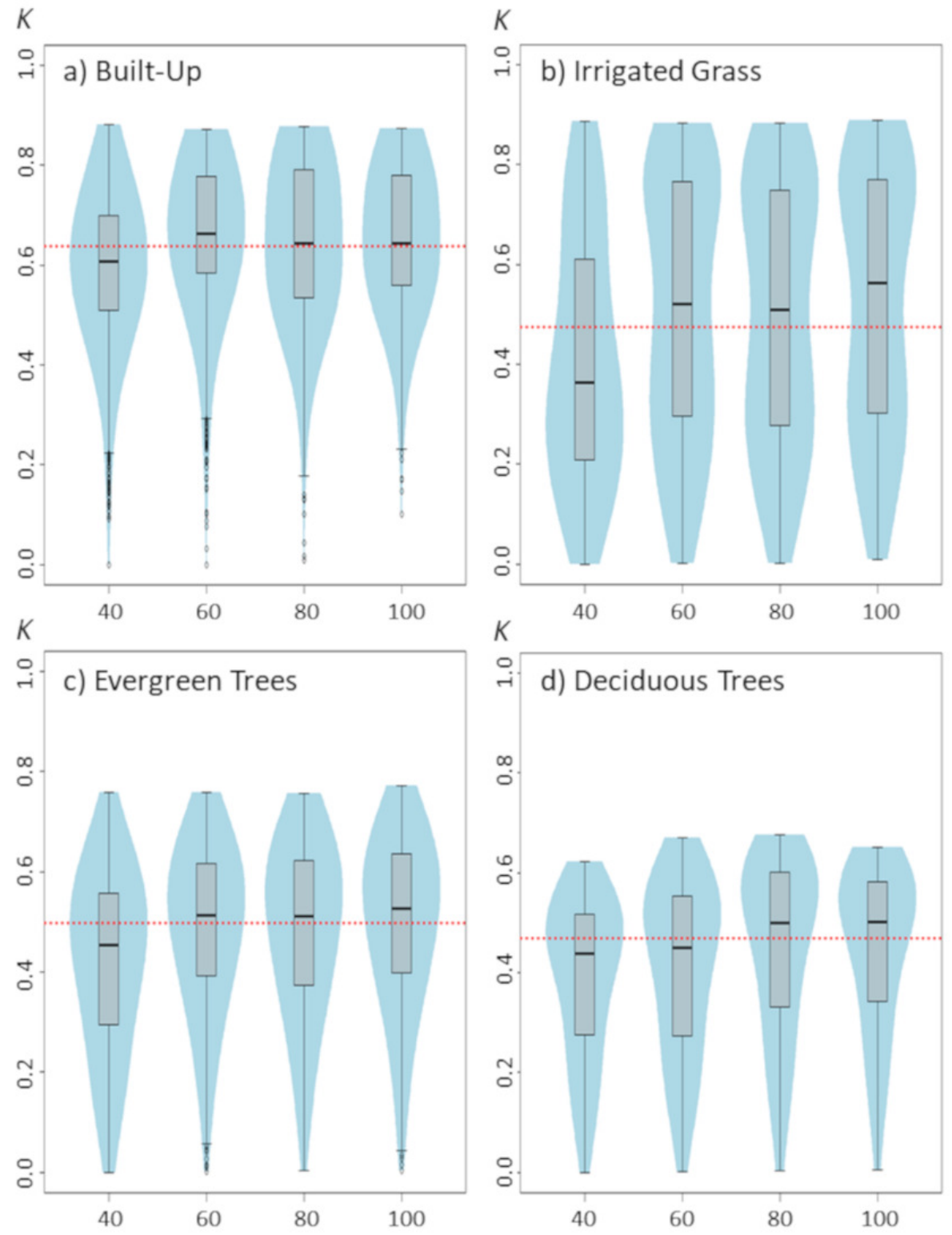
Figure 4

Violinplots of the kappa values obtained from the single land-cover maps generated through MaxEnt using the test percentage (TP) shown in the $x$-axis.

Each violinplot includes the combination of all the other tested parameters (See Table 1 for reference). Red dashed line is the median kappa value for all combinations. 

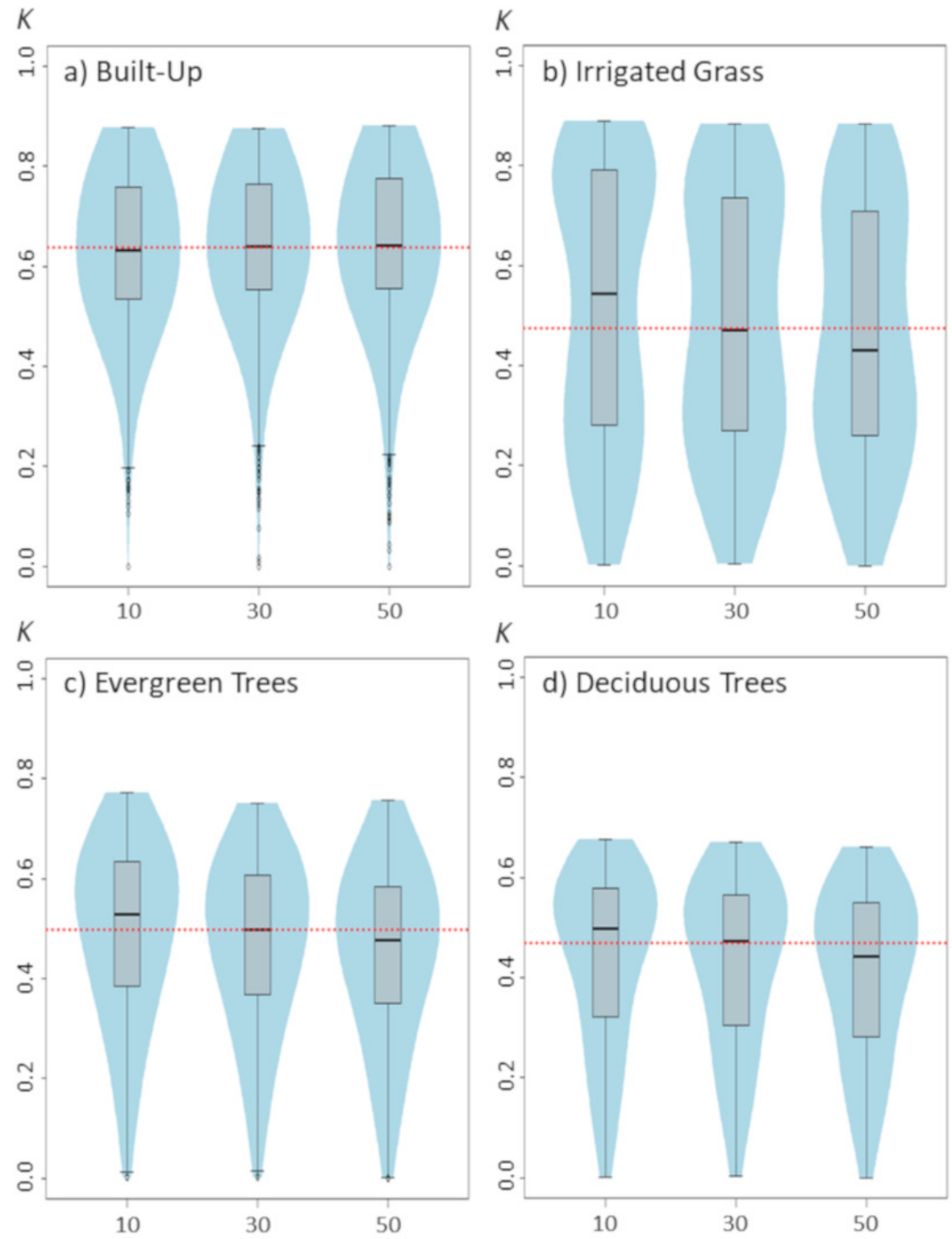
Figure 5

Violinplots of the kappa values obtained from the single land-cover maps generated through MaxEnt using the binary threshold (BT) shown in the $\mathrm{x}$-axis.

Each violinplot includes the combination of all the other tested parameters (See Table 1 for reference). Red dashed line is the median kappa value for all combinations.
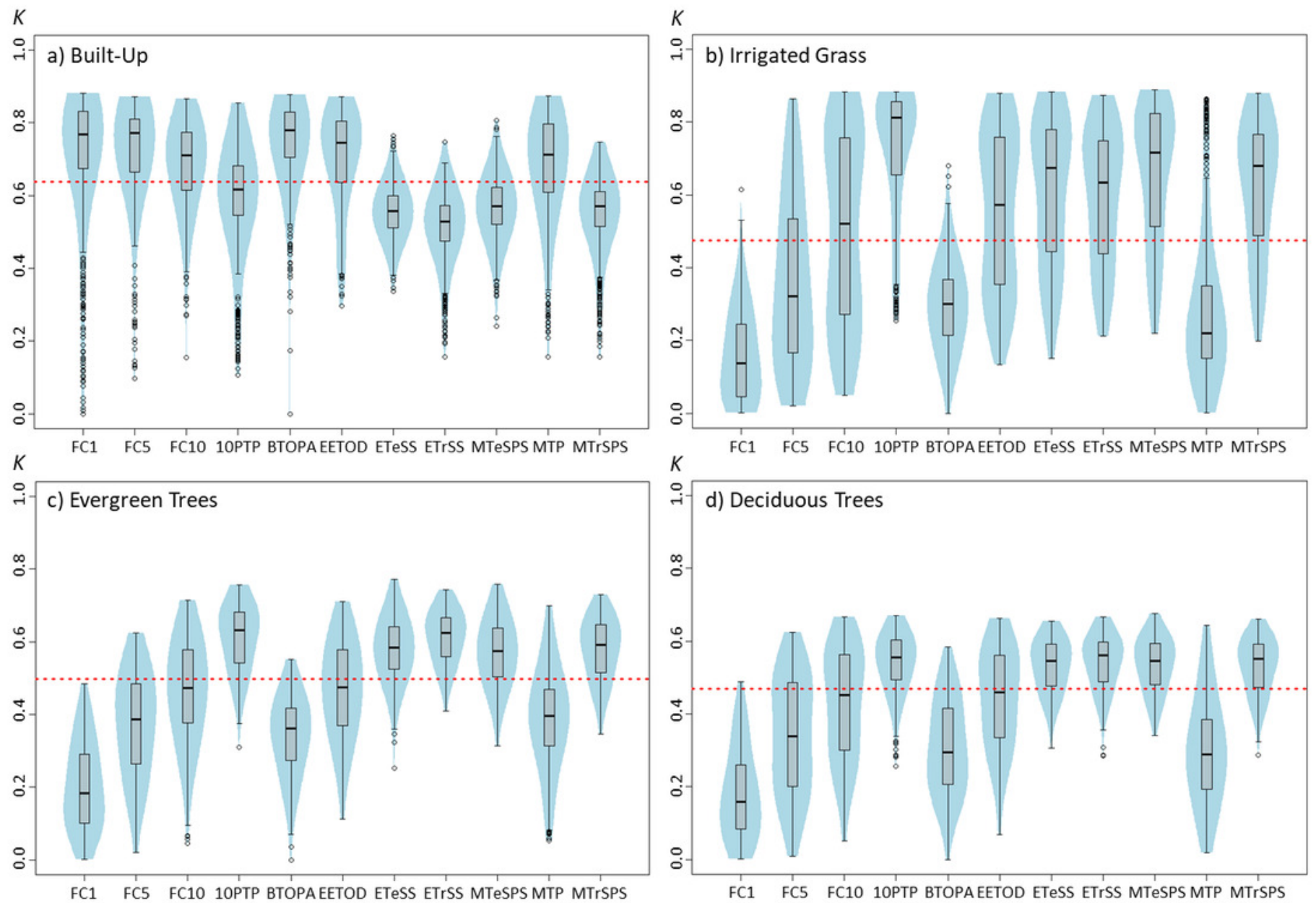


\section{Figure 6}

Barplots comparing the average kappa values obtained from the single land-cover maps generated through MaxEnt using the default vs best parameters settings.

Bars show the mean $( \pm \mathrm{SE})$ kappa value of 30 replicates after applying the $11 \mathrm{BT}$. All models were built using a SS of 100 and a TP of $10 \%$. The best FC and RM parameter setting used for each land-cover type is shown in the figure legend. Statistically significant values are represented by $\mathrm{p}$-values of $*(<0.05), * *(<0.01), * * *(<0.001), * * * *(<0.0001)$, after applying a two-sided t-test for each comparison (i.e. default vs best parameter setting).
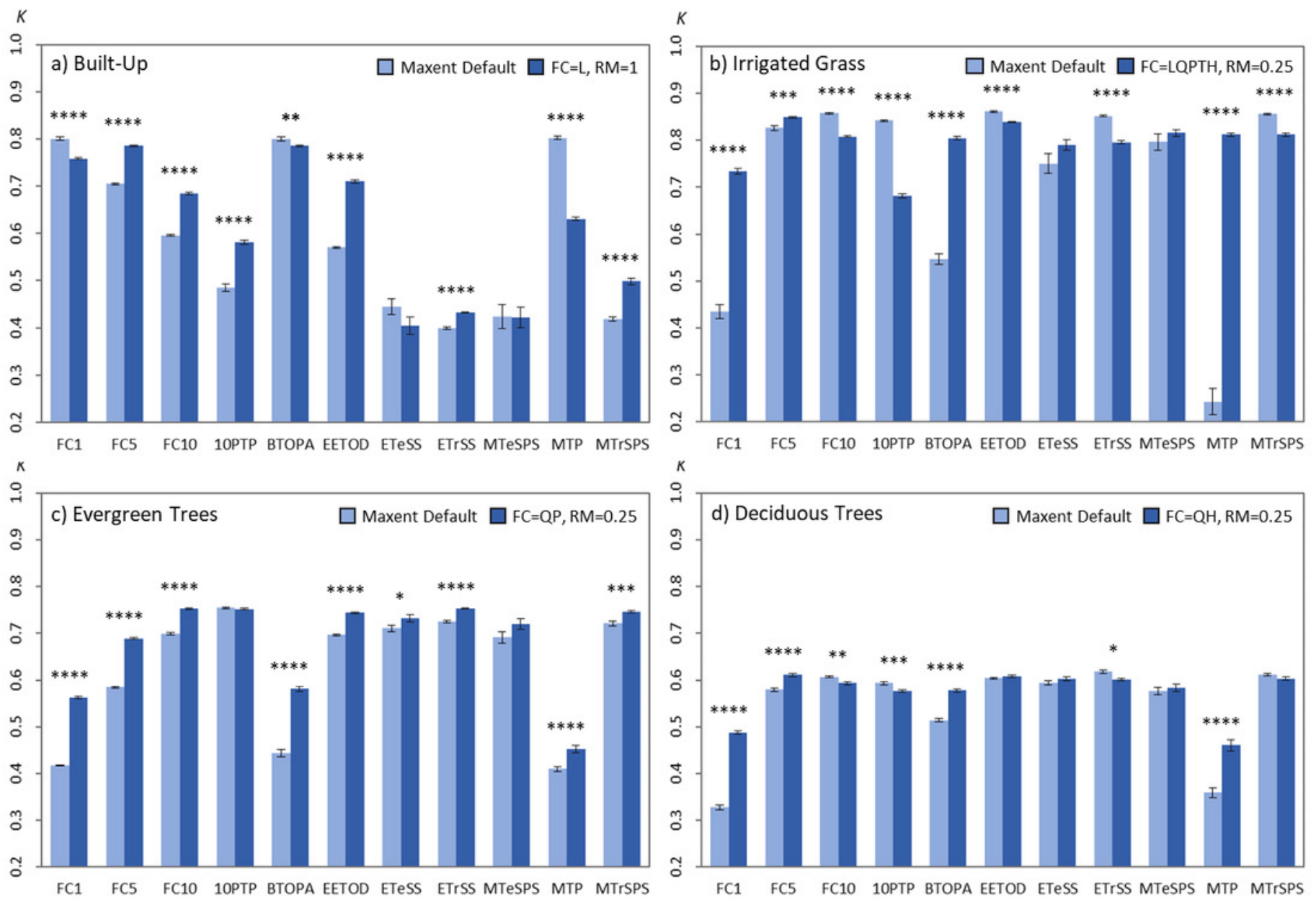